\title{
Game Analysis of Anti-Corruption of Government from the Perspective of Interest Group
}

\author{
Yu Zhang \\ School of Public Administration of Shanxi University of Finance and Economics, Taiyuan, Shanxi \\ 030006
}

\begin{abstract}
Purpose: In recent years, anti-corruption has been continuously strengthened, and various corruptions appear gradually. The purpose of this paper is to find out solutions of reducing anti-corruption costs and enhancing corruption costs so as to reduce corruption phenomena. Methods: the research in this paper, based on rational economic man's assumption, is to study costs and benefits of corruptions among people in government departments and also costs and benefits of China anti-corruption from the perspective of interest groups by building a cost-benefit model and double-person game model. Conclusion: cost-benefit analysis and behavior game indicates that it is the best way to strike corruptions by enhancing corruption costs and reducing anti-corruption costs in face of the problems of corruption among government officials.
\end{abstract}

Keywords: Interest group; Government; Costs; Benefits; Game.

\section{利益集团视角下政府反腐行为的博弯分析}

\author{
张瑜 \\ (山西财经大学公共管理学院, 山西太原, 030006)
}

摘要: 目的: 近几年我国的反腐力度不断增加, 各种腐败行为浮出水面。本文的目的是寻找降低反腐败成本、提高腐败 成本的解决措施, 来降低腐败现象。方法: 本文的研究是在理性经济人假设的基础上, 在利益集团的视角之下, 通过建立成 本一收益模型以及双人博弯模型, 对政府部门的人员腐败的成本和收益进行研究, 以及对国家反腐败的成本和收益的进行 研究。结论: 从成本收益分析和行为博弯可知, 面对政府官员的腐败问题, 只有提高腐败成本、降低反腐败的成本, 是打击 腐败的最好办法。

关键词: 利益集团；政府；成本；收益；博弃

\section{引言}

自党的十八大以来，习近平总书记强调要 “苍蝇”、“老虎”一起打，党中央重拳打击反腐，国家的 反腐力度越来越大, 中央巡视组入驻各层级政府以及有关部门进行调查, 取得的反腐成绩也是非常突出的, 反腐已经成为一种新常态。[1]但从另外一个方面也反映出我国腐败的严重程度。“透明国际” [2]组织每 年都会发布各个国家的清廉指数，2015 年，在被评估的 168 个国家和地区中，中国大陆排名第 83 名。我 国腐败现象的发展势头迅猛，腐败程度已经危及和破坏法律的权威性和有效实施，甚至破坏了我国社会主 义的经济基础，动摇着我国社会的政治基础。腐败问题已经对党，对国家和社会构成了潜在的威胁。政府 反腐力度的增加确实可以给予腐败人员一定的威慑力, 但是只有真正理解腐败产生的原因, 才能更好地解 决腐败问题。

\section{1 相关概念界定}

利益集团是指持有共同态度、向社会其他集团提出要求的松散或严密的组织。如果它通过向政府的任 
何机构提出其要求, 它就变成一个政治性的利益集团。政治性的利益集团就是这样一个组织, 它使用各种 途径和方法向政府施加影响, 进行非选举性的鼓动和宣传, 用以促进或阻止某方面公共政策的改变, 以便 在公共政策的决策中，体现自己的利益主张。本文中提到的利益集团均指政治性的利益集团。政府利益可 以是政府所代表的公共利益，也可以是政府自身源于权力的利益，以及政府部门职员自身的利益，本文只 研究政府部门的职员个人利益。

本文所有行为主体的行为都是基于理性经济人假设, 是追求自身利益最大化的。利益集团的组成目的 就是追求利益最大化, 体制内的切分以及体制外的追逐, 并且管理成本、机会成本或交易成本都要最小化, 即便是犯罪成本都要最小化。又根据政府人员自身 “理性经济人” 的性质, 政府是无法真正成为一个中性 的、契约的政府，稍有不慎，政府就容易变质为 “掠夺的政府” ——腐败的政府，即政府人员利用职务之 便来获取个人利益。由于政府的以上性质，政府是一个特殊的利益集团。

由于政府及其权力的膨胀是源于对利益的追求，中央与地方政府的博竕是源于政府利益的存在，“上 有政策, 下有对策” 现象严重, 利用权力进行利益转移和掠夺这几点表明, 政府的行为带上了利益的烙印。 [3]其他利益集团为政府提供丰富的决策信息，使政府决策更好地反映民意、服务民众；它的活动在一定 程度上对政府有监督、监察作用，有助于政府决策的公正性、合理性; 人们通过利益集团来表达自己的观 点、意见, 追求自己的利益, 这在一定意义上也是实践民主政治的一种有效形式。但是, 我们也看到中国 现存的很多顽症: 腐败问题、地方保护、变相地方债 (集资)、地方关税、体制改革、信用恶化、坏账问题、 行业垄断、价格失效、豆腐渣工程等等大多跟利益集团有关。

\section{2 政府腐败的产生与现状}

改革开放 30 年来，我国正从一个同质的单一性社会向异质的多样性社会转型。在社会转型过程中， 整个社会利益结构发生了分化与重组，社会阶层构成发生了新的变化，社会上出现了诸如私营企业主、个 体户、自由职业人员、受聘受雇人员等不同的利益群体和利益阶层。在实践中，政府部门的腐败很多都是 集体或者团体腐败而非个人腐败。新的利益群体和利益阶层逐步分化组合成特定的 “利益集团” ，在此基 础上，加之公民利益意识的觉醒和维权意识的加强，特别是 “集团意识” 的形成，以及政治的民主化、法 治化和现代交通与信息技术的发展，使得我国利益集团的产生成为一种客观趋势。[4] 随着市场化的发展 以及政府拥有广泛资源与强大干预能力, 中国的各种 “利益集团” 对政府的决策以及政策的执行施加广泛 而深远的影响，政府便出现了腐败。

一是众多境外与涉外利益集团对政府人员进行行贿。如各国在华商会以及中国外商投资企业协会 (商 务部下属的行业协会), 通过母国政府或政治游说, 或收买、利用高干子女、亲属, 或聘请一些部门领导 与职员做咨询师 (拿咨询费), 或将相关课题并配以丰厚的课题经费, [5] 给予各大部委研究机构与学者等 途径，对我相关部门决策与立法施加巨大影响，致使我大量政经信息外泄。

二是金融、能源、邮电、运输、基础建设等领域的国有垄断企业，长期依托行政垄断，拥有强大的博 亦能力。为维护龙头地位、持续获得垄断利润, 有些垄断企业在政界、学界、传媒界网罗代言人, 影响甚 至操纵话语权, 为其垄断地位辩护, 极力排斥行业竞争与民营经济介入, 抵制《反垄断法》等于己不利的 法律政策出台, [6]或以本行业的特殊情况 (如自然垄断、国家安全、为政府赚钱等) 为由要求从相关法律 政策中得到豁免, 维持垄断。 
三是一些民营企业家争当人大代表或政协委员，利用人大与政协这两大政治舞台，来声张、谋取其利 益; 或私下聘请相关专家学者担任独立董事, 为其摇旗呐喊; 或通过行贿等不正当的利益输送直接影响行 政决策。自中央推行宏观调控抑制房地产价格过快增长的政策出台后，有的房地产开发商迅即与一些专家 学者、房地产研究机构、部分媒体、甚至官员联手, 强占行业话语制高点, 并形成一个行业性的 “话语链”, 以对抗宏观调控。在实践中, 政府部门的腐败很多都是集体或者团体腐败而非个人腐败。

可见，一些政府同强势集团利益合流。我国利益集团目前最活跃的当属一些经济利益集团，他们通过 自己的一些行动来影响政府, 从而获取了巨大利益, 甚至是以损害其他利益集团利益为基础。而政府的作 用不是维护合理的竞争，而是在其中获取了巨大利益，甚至与强势利益集团形成利益合流；政府在对待不 同利益集团的态度不同, 有失公正; 一般说来, 在经济发展中起到较大作用的利益集团, 政府对他们采取 听从的态度，而对在改革中利益相对较弱的集团却采取一定的压制手段。

\section{3 政府腐败产生的原因分析}

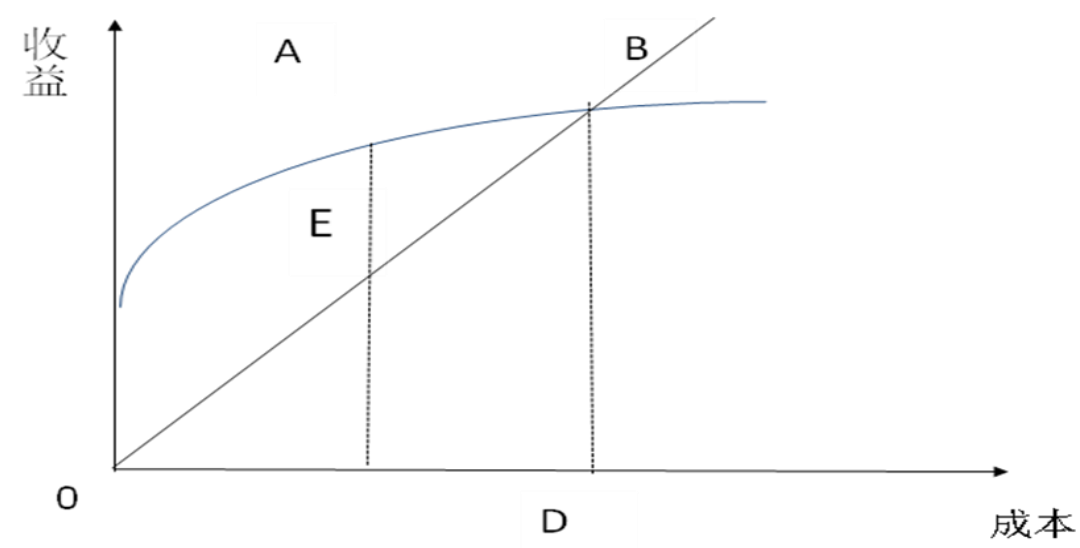

图 1 利益集团贿赂行为的成本-收益模型

认清政府腐败的原因，是根治或者减少腐败的根本。[7]如图所示，曲线代表利益集团的成本一一收 益曲线, 即利益集团为了得到一定的收益, 他必须花费一定的成本, 而这成本就是贿赂政府人员, 从曲线 的趋势可以看出, 随着对政府的贿赂成本的增加, 利益集团的收益是先快速增加后增加的趋势变缓; 斜度 为 $45^{\circ}$ 角的直线代表成本和收益相等, 即利益集团贿赂政府人员的分界线, 当利益集团的收益在分界线之 右下方或这条线上时, 利益集团是不会进行贿赂的, 而在分界线之左上方和成本一一收益曲线所组成的区 域里, 利益集团就会对政府人员进行贿赂。可见, 只要利益集团付出成本后获得的利益很高, 那么他们对 政府人员进行贿赂就具有很高的积极性。

政府人员在面对利益集团的贿赂时，是否会做出腐败行为决定于假设他们做出该行为之后，他们对所 付出的成本和所获得收益之间的衡量和比较。腐败成本是指腐败行为人因实施腐败行为所承受的精神性、 物质性代价，是一种成本支出。[8] 政府人员面对的腐败交易成本包括以下方面：腐败的经济成本、腐败 的政治成本以及腐败的道德成本。腐败收益是指政府腐败人员通过权钱交易谋取的私利, 也可以说是腐败 成本投入之后的 “产出” 除去成本之后, 腐败行为人所得到的 “收益”。这种收益绝非仅指经济上的利益, 还包括对财物占有欲望的满足。当个人腐败收益大于成本时, 作为一个追求自身利益最大化的理性人，腐 败是一个理性博亦之后的最佳选择。下面我们来建立腐败行为的决策博弯, 假设 A、B 是两个同为拥有一 
定权力的政府人员, 我们对其 A、B 在面对贿赂的时候他们可能做出的行为进行博弯分析, 结果如表 1 所 示。

表 1 政府人员面对贿赂的双人博弯

\begin{tabular}{lllr|rr}
\hline \hline & 行为 & \multicolumn{3}{c}{ B } \\
\cline { 3 - 6 } & & \multicolumn{3}{c}{ 接受贿赂 } & \multicolumn{1}{c}{ 不接受贿赂 } \\
\hline $\mathrm{A}$ & 接受贿赂 & & 5 & & -1 \\
& & 5 & & 7 & \\
\cline { 3 - 6 } & & & & & 2 \\
\hline \hline
\end{tabular}

在我国，政府人员的腐败行为所花费的经济成本很少，法定惩罚成本不高，心理承受成本不高并且低 风险。这个时候接受贿赂的收益就大于不接受贿赂的收益。当每个人都追求自身利益最大化时, A、B二人 一起腐败是理性的选择, 因此政府人员腐败是必然的。从表中我们可以看出, A、B 同时接受贿赂是最好的 选择。腐败是一种低风险、高回报的经济行为，任何理性人都会觉得值得投资。

从根本上说，我国这一时段的腐败滥解于经济对政治的支配，即政治性的利益集团对官僚集团的收买 和控制。在中国转型过程中, 由于资金、土地、劳动力等主要生产要素仍由行政力量决定和配置, 形成了 行政配置手段与市场配置手段并行的双轨制, 从而产生了一个巨大的租金空间, 滋生、诱发了以权力寻租 为主的腐败犯罪。[9]它们之间的关系类似于经济学中的 “委托一代理” 关系, 政府之所以成为被俘获的 对象, 主要是因为它手中握有大量公共资源, 其中包括财政预算支出、诸如土地和矿产等公共财产、非中 性的法律规章制度的设立等。同时, 运转政府的公职人员在分配公共资源的过程中拥有一定的自由裁量权, 直接导致腐败。

\section{4 降低政府官员腐败的措施}

从成本收益分析和行为博弯可知，面对政府官员的腐败问题，只有提高腐败成本、降低反腐败的成本， 是打击腐败的最好办法。

提高腐败成本。加强法律打击力, 并提高腐败侦破效度。打击腐败作为一种惩罚成本, 是运用国家强 制力迫使一切铤而走险者就范, 在侦破、定罪和量刑这一打击循环圈的各个环节中, 定罪关系到事实和法 律, 主观和客观, 既不能定义过宽, 也不得主客观相违背; [10]量刑的局限在于生命刑作为成本不是也不 可能是可以无限增长的, 故侦破的效度就成为加大打击力度的主要环节, 因此有效的打击, 必须重视侦破 率的提高。

降低反腐败成本。加强與论媒体监督、人民群众的监督以及利益集团的监督。首先新闻媒体界对一些 事件的报道, 是十分有效的管理工具, 可协助商业机构和政府在事态转趋严重前, 及早纠正错失。在遏制 腐败的过程中, 通常是由新闻媒体对腐败事件或腐败人物的披露为先导; 其次, 鼓励人民群众行使自己的 與论监督权, 可以一举改变 “少数人监督多数人” 的宕境, 由人民群众实施多数人对少数人的监督, “搞 歪门邪道者无论多么有 “实力’ , 总是害怕阳光, 不敢面对大众。在现代社会, 只要把事情端到台面上, 问题的公正解决就有了一半的可能” ; 最后, 势均力敌的利益集团之间出现利益冲突并形成的相互制衡与 监督, 客观上也可以成为抑制腐败的有效途径之一。通常意义上讲, 现行腐败体制的既得利益者不会生发 
变革现状的需求或动机。真正意义上的挑战者来自那些不能从现行制度中受益、却试图以改革为旗号重新 确立权力分配机制的利益集团。政治上的竞争以及支配政治竞争的强大利益集团，均产生了以腐败罪名指 控反对派或潜在挑战者的动机，从而在客观上提高了隐蔽性腐败行为被曝光的可能性。与此同时，政治利 益的冲突也会激励法官严厉处罚政治对手构成犯罪的腐败行为。同时，这些监督可以说几乎不花一分钱， 是反腐败过程中成本最低的“社会防腐剂”!

当然，也要加大反腐败的必要投入，加强对反腐倡廉的教育宣传力度。治贪必须先育人，而作为对人 具有潜移默化内在功能的教育手段，成为预防腐败最基本、最广泛和最有效的措施。具体的教育手段有: 一是加强廉政教育, 创造一种从政廉洁、为官清廉的社会风气; 二是加强道德教育, 开展以社会公德、职 业道德和家庭美德为内容的公共道德建设活动; 三是加强法纪教育, 营造一个人人知法、守法并憎恶违法 的良好社会环境。

\section{6 致谢}

本文系山西省哲学社会科学规划课题（晋规办字 [2015]3 号)、山西财经大学青年科研基金项目 （QN-2014014）的阶段性成果。

\section{参考文献:}

[1] 徐喜林，徐栋．法治反腐:中国反腐新常态 [J]．中州学刊，2015，No. 218(2):15-18.

[2] 透明国际 (Transparency International) 即 “国际透明组织”，简称 TI，是一个非政府、非盈利、国际性的民间 组织。今天, “透明国际” 已成为对腐败问题研究得最权威、最全面和最准确的国际性非政府组织, 目前已在 120 个国家成 立了分会。它的研究结果经常被其他权威国际机构反复引用。

[3] 李燕凌，吴松江，胡扬名．我国近年来反腐败问题研究综述 [J]．中国行政管理，2011 (11) :115-119.

[4] 陈刚. 上行下效:高官腐败的示范效应研究 $[J]$. 经济社会体制比较, 2013(2):155-164.

[5] 公婷, 吴木較. 我国 2000-2009 年腐败案例研究报告——基于 2800 余个报道案例的分析 [J]. 社会学研究, 2012 (4) : 204-220.

[6] 陈国权，毛益民. 腐败裂变式扩散：一种社会交换分析 [J]. 浙江大学学报人文社会科学版，2013，43(2):5-13.

[7] 何家弘. 中国腐败犯罪的原因分析 [J]. 法学评论, 2015 (1):68-76.

[8] 过勇. 当前我国腐败与反腐败的六个发展趋势 [J]. 中国行政管理, 2013(1):60-63.

[9] 熊光清. 当前中国的腐败问题与反腐败策略 [J]. 社会科学研究, 2011 (5) : 53-58.

[10］文宏，黄之玞. 网络反腐事件中的政府回应及其影响因素——基于 170 个网络反腐案例的实证分析 $[\mathrm{J}]$. 公共管 理学报, 2016(1):21-30.

\section{References}

[1] $\mathrm{Xu}$ Xilin, $\mathrm{Xu}$ Dong. Legal Anti-Corruption: China's Anti-Corruption New Normal [J]. Academic journal of Zhongzhou, 2015, No. 218(2):15-18.

[2] Transparency International (short for TI) is "International Transparency Organization", which is a non-government, non-profit, international civil organization. Today, "Transparency International" has become an international non-government organization which has done the most authorized, thorough, and precise research on the corruption issues. At present, it has set up chapters in 120 countries. Its research results are often quoted repeatedly by other authorized international institutions.

[3] Li Yanling, Wu Songjiang, Hu Yangming. Research Summary about China's Anti-Corruption Problems in Recent years [J]. Chinese Public Administration, 2011(11):115-119. 
[4] Chen Gang. People follow the Example of Their Superiors: Analysis of the Example Effect of Senior officers' Corruption [J]. Comparative Economic \& Social Systems, 2013(2):155-164.

[5] Gong Ting, Wu Muluan. Analysis Report on Corruption Case During 2000-2009 in China-Analysis Based on over 2800 Reported Cases [J]. Sociological Studies, 2012(4):204-220.

[6] Chen Guoquan, Mao Yimin. Fission Diffusion of Corruption: A kind of Social Exchange Analysis [J]. Journal of Zhejiang University (Humanities and Social Science), 2013, 43(2):5-13.

[7] He Jiahong. Cause Analysis on China's Corruption Crimes [J]. Law Review, 2015(1):68-76.

[8] Guo Yong. 6 Development Trends of Current Corruption and Anti-Corruption in China [J]. Chinese Public Administration, 2013(1):60-63.

[9] Xiong Guangqing. Current Corruption Problems and Anti-Corruption Strategies in China [J]. Sociological Studies, 2011(5):53-58.

[10] Wen Hong, Huang Zhifu. Government Respond to Network Anti-Corruption Event and Its Influencing Factor-Based on Empirical Analysis of 170 Network Anti-Corruption Cases [J]. Journal of Public Management, 2016(1):21-30 